\title{
Identification of a novel polyprenylated acylphloroglucinol-derived SIRT1 inhibitor with cancer-specific anti-proliferative and invasion-suppressing activities
}

\author{
LIJIA ZHU ${ }^{1}$, JI QI ${ }^{2}$, CHRISTINE YA-CHI CHIAO ${ }^{1}$, QIANG ZHANG ${ }^{2}$, JOHN A. PORCO Jr ${ }^{2}$, \\ DOUGLAS V. FALLER ${ }^{1}$ and YAN DAI ${ }^{1}$ \\ ${ }^{1}$ Cancer Research Center and Department of Medicine, Boston University School of Medicine, Boston, MA 02118; \\ ${ }^{2}$ Department of Chemistry, Center for Chemical Methodology and Library Development (CMLD-BU), \\ Boston University, Boston, MA 02215, USA
}

Received May 23, 2014; Accepted July 10, 2014

DOI: 10.3892/ijo.2014.2639

\begin{abstract}
SIRT1, a class III histone deacetylase, plays a critical role in regulating cancer cell growth, migration and invasion, which makes it a potential target for cancer therapeutics. In this study, we screened derivatives of several groups of natural products and identified a novel SIRT1 inhibitor JQ-101, a synthetic derivative of the polyprenylated acylphloroglucinol (PPAP) natural products, with an $\mathrm{IC}_{50}$ for SIRT1 of $30 \mu \mathrm{M}$ in vitro, with 5-fold higher activity for SIRT1 vs. SIRT2. Exposure of tumor cells to JQ-101 significantly enhanced acetylation of p53 and histone H4K16 at known sites of SIRT1 deacetylation, validating SIRT1 as its cellular target. JQ-101 suppressed cancer cell growth and survival by targeting SIRT1, and also exhibited selective cytotoxicity towards a panel of human tumor cell lines, while producing no toxicity in two normal human cell types at comparable concentrations. JQ-101 induced both apoptosis and cell senescence, and suppressed cancer cell invasion in vitro. In summary, we have identified JQ-101 as a new SIRT1 inhibitor which may have potential application in cancer treatment through its ability to induce tumor cell apoptosis and senescence and suppress cancer cell invasion.
\end{abstract}

\section{Introduction}

SIRT1 is a nicotinamide adenine dinucleotide (NAD)dependent histone deacetylase that has been reported to play an important role in a variety of physiological processes including

Correspondence to: Professor Yan Dai, Cancer Center, Boston University School of Medicine, 72 E. Concord St, L913, Boston, MA 02118, USA

E-mail: yandai@bu.edu

Key words: SIRT1 inhibitor, polyprenylated acylphloroglucinol, cancer cell growth, cancer cell invasion aging, metabolism, cell survival and carcinogenesis (1-6). Moreover, there is growing evidence strongly suggesting that SIRT1 has a role in cancer progression (7-14). SIRT1 suppresses apoptosis and cell senescence and also promotes cell migration and invasion (14-18), angiogenesis $(19,20)$, and drug resistance in multiple models (7-10). In vivo studies show that SIRT1 inhibition suppresses tumor growth, metastasis and progression in several cancer types, including prostate, breast and neuroblastoma $(11,12,14)$. In addition, SIRT1 overexpression has been shown to correlate with poor prognosis in several cancer types, including large B-cell lymphoma (21), prostate cancer $(18,22)$, pancreatic cancer $(23)$, gastric cancer $(24,25)$, breast cancer (26), hepatocellular carcinoma (27), colorectal cancer (28) and lung cancer (29). Collectively, these results suggest an important role for SIRT1 in cancer growth and progression. SIRT1 inhibitors are therefore of significant interest as potential therapeutic agents.

Several inhibitors of SIRT1 have been reported, including nicotinamide (30), sirtinol (31), cambinol (32), EX-527 (33), Tenovin-6 (34), splitomycin (35), toxoflavin (36), salermide (37), 2-anilinobenzamides (38) among other compounds. These SIRT1 inhibitors can induce selective cytotoxicity in cancer cells in vitro $(31,32,34-36,38,39)$. In addition, several SIRT1 inhibitors have been tested in cancer xenograft mouse models $(32,34,40)$. Cambinol was well tolerated in mice and significantly inhibited the growth of Burkitt lymphoma xenografts (32). Tenovin-6 suppressed tumorigenesis of melanoma and N-Myc-induced neuroblastoma (34), and inauhzin, a phenothiazine, reduced colon xenograft growth (40). These results provide proof-of-concept examples that SIRT1 inhibition may be an effective modality in cancer therapy.

Here we report the identification of a new SIRT1 inhibitor, JQ-101, which induces cancer cell apoptosis and senescence, suppresses cancer cell invasion, and exerts cancer-specific cytotoxity, repressing tumor cell growth.

\section{Materials and methods}

Cells, antibodies and reagents. All cancer and normal cells lines were obtained from the American Type Culture 
Collection (Manassas, VA). LNCaP, PC3, Ramos, Jurkat, H1299 and MRC5 cells were maintained in RPMI-1640 medium with 10\% FBS (HyClone, CO). H460, A549, ZR75 and MDA231 cells were maintained in DMEM medium with $10 \%$ FBS. PZ-HPV-7 cells were maintained in Keratinocyte Serum-Free Medium supplemented with Epidermal Growth Factor (Invitrogen, Carlsbad, CA).

Antibodies to SIRT1 (sc-74504) were purchased from Santa Cruz Biotechnology (Santa Cruz, CA). Antibodies to Ac-p53, p53, Ac-Histone H4 and H4 were purchased from Millipore (Billerica, MA). Antibodies to $\beta$-actin were purchased from Sigma-Aldrich (St. Louis, MO). Sirtinol was purchased from Sigma-Aldrich.

Chemical synthesis of polyprenylated acylphloroglucinol (PPAP) analogues. JQ-101, JQ-2, JQ-3, JQ-4, JQ-5,JQ-6, JQ-7, JQ-8, JQ-9, JQ-10, JQ-11, JQ-31, JQ-32, JQ-33 and JQ-34 (Fig. 1) are simplified analogues of the type B PPAP natural product clusianone and were synthesized using our reported procedure involving tandem alkylative dearomatization-annulation of acylphloroglucinols to rapidly construct the bicyclo[3.3.1] nonane-1,3,5-trione core (41). BM001, BM002, BM003, BM004, BM005, BM006, BM007, ВM008, BM01810, BM01817, BM01847, BM-01-1005, BM-01-1013F2, BM-01-1011, BM-01-1022 and related bicyclo[2.2.2] octadiones (Table I) were synthesized using the reported method involving $\mathrm{Mn}(\mathrm{III}) / \mathrm{Cu}(\mathrm{II})$-mediated oxidative radical cyclizations of dearomatized phloroglucinol substrates (42). Compounds QZ-2001-2005, analogues of the type A PPAP nemorosone, were prepared as intermediates during the course of our chemical synthesis of 7-epi-nemorosone (43).

SIRT1 and SIRT2 activity analysis and small molecule screening. The Cayman hSIRT1 activity assay kit (SIRT1 Direct Fluorescent Screening Assay Kit, cat. no. 10010401) and hSIRT2 activity assay kit (SIRT2 Direct Fluorescent Screening Assay Kit, cat. no. 700280) were used to quantitate the $\mathrm{IC}_{50} \mathrm{~S}$ of the SIRT inhibitors. The assay was carried out according to the manufacturer's instructions. All compounds were preincubated with the hSIRT1/hSIRT2 proteins before commencing the reaction through the addition of the 'Fluor de Lys' deacetylase substrate. Deacetylation of K382-p53/K320-p53 was used as a marker of HDAC activity. Fluorescence was read (Ex 360 nm/Em 460 nm) using Synergy HT Multi-Mode Microplate Reader (BioTek). Assays were repeated in triplicate. Quantitation of acetylation was derived from the levels of fluorescence (displayed in arbitrary fluorescence units), and the percentages of inhibition were calculated from the arbitrary fluorescence unit of the treated assays and non-treated controls. The $\mathrm{IC}_{50}$ was determined using GraphPad Prism 6.0.

Establishment of stable SIRT1-knockdown cell lines. The Phoenix packaging cell line was transfected with shRNA expression plasmids pSUPER.retro.puro-SIRT1 (5'-GATGAA GTTGACCTCCTCA-3') or pSUPER.retro.puro-non-targeting shRNA control separately, using Lipofectamine 2000 (Invitrogen). After $48 \mathrm{~h}$, the medium containing retrovirus was collected, filtered, treated with polybrene and transferred to LNCap or H460 cell cultures. Infected cells were selected with puromycin and the stably-infected colonies were pooled.
Table I. Cytotoxicity measurement of JQ-101 in multiple cancer/normal cell lines.

\begin{tabular}{lc}
\hline Cancer cell lines & $\mathrm{IC}_{50}(\mu \mathrm{M})$ \\
\hline LNCaP & 20 \\
Jurkat & 25 \\
H460 & 30 \\
Ramos & 33 \\
A549 & 40 \\
MDA-MB-231 & 60 \\
ZR75 & 66 \\
H1299 & 85 \\
PC3 & 119 \\
Normal cell lines & \\
PZ-HPV-7 & 250 \\
MRC5 & 300 \\
\hline
\end{tabular}

Immunoblot analysis. Cells were lysed with $1 \%$ Nonidet P-40 lysis buffer (1\% NP-40, $150 \mathrm{mM} \mathrm{NaCl,} 50 \mathrm{mM}$ Tris- $\mathrm{HCl}$ ) with protease inhibitor (Roche Diagnostics). Protein samples were subsequently separated on an $8 \%$ SDS-polyacrylamide gel and analyzed with anti-Ac-p53, anti-p53, anti-SIRT1, anti-AcHistone $\mathrm{H} 4$, anti-H4 or $\beta$-actin antibodies.

Cell viability assay. MTS (Promega, G3580) assays were used to quantify cell viability. Cells were plated at a density of $10^{4}$ cells/well on 96-well plates and exposed to various concentrations of JQ-101, ranging from 5 to $125 \mu \mathrm{M}$, for $72 \mathrm{~h}$. A total of $20 \mu \mathrm{l}$ MTS CellTiter96Aqueous one solution reagent was added to the medium for $1 \mathrm{~h}$, and the absorbance was read at $490 \mathrm{~nm}$.

Apoptosis analysis by FACS. The cultured cells were trypsinized, collected and labeled with Annexin V antibody and PI. The procedure for cell labeling followed the Annexin V kit instructions (Annexin V-PE Apoptosis Detection Kit I, BC Pharmingen, cat. no. 559763). The labeled samples were further analyzed with flow cytometry.

SA- $\beta$-gal staining. The cells were seeded at $30 \%$ confluence, and JQ-101 was added to a final concentration $50 \mu \mathrm{M}$. At 4 days after the addition of JQ-101 to the culture media, the cells were washed with phosphate-buffered saline (PBS) and fixed with PBS containing $2 \%$ formaldehyde and $0.2 \%$ glutaraldehyde for $10 \mathrm{~min}$. The cells were then incubated at $37^{\circ} \mathrm{C}$ overnight with staining solution $[40 \mathrm{mM}$ citric acid sodium phosphate, $\mathrm{pH} 6.0,1 \mathrm{mg} / \mathrm{ml}$ 5-bromo-4-chloro-3-isolyl- $\beta$-D-galactoside (X-gal, Fisher, Pittsburgh, PA), $5 \mathrm{mM}$ potassium ferricyanide, $150 \mathrm{mM} \mathrm{NaCl}, 2 \mathrm{mM} \mathrm{MgCl} \mathrm{M}_{2}$. SA- $\beta$-gal-positive cells were enumerated by microscopy.

Cell invasion assay. Cell invasion activity of cancer cells was assessed using BD BioCoat Matrigel Invasion Chambers (BD cat. no. 354480). Briefly, the cells were trypsined, washed with PBS, and resuspended in media containing $1 \%$ FBS at a density of $5 \times 10^{4}$ cells $/ \mathrm{ml}$. A total of $500 \mu \mathrm{l}$ of cells suspended in medium containing JQ-101 or DMSO (vehicle control) was 


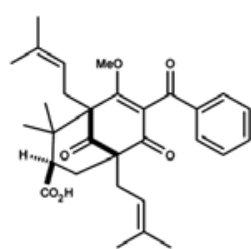

JQ-101

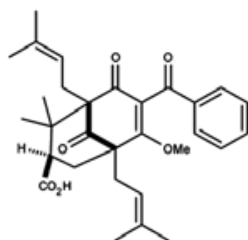

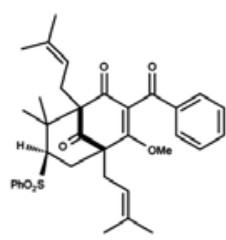

JQ-06

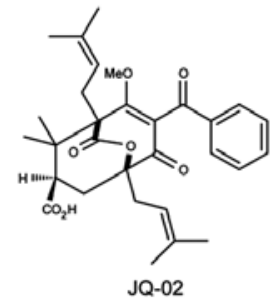

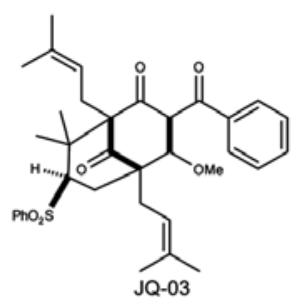

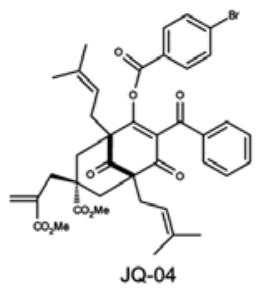

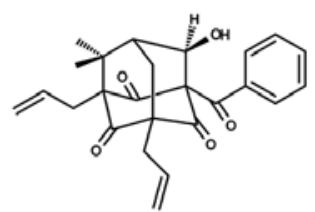

JQ-07

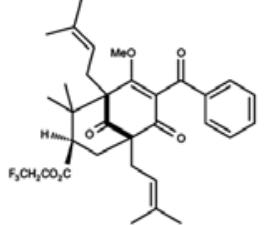

JQ-08

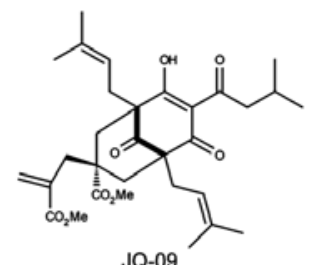

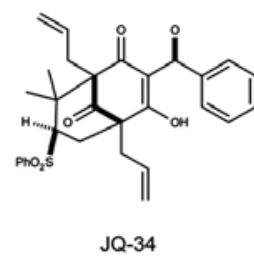<smiles>C=CC[C@]1(C)C(=O)C(CC=C(C)C)=C(O)C(CC=C(C)C)(CC=C(C)C)C1=O</smiles>

BM001

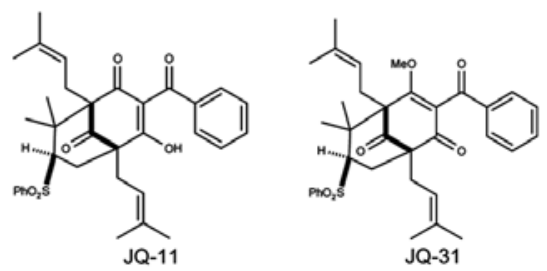<smiles>C=CCC12C(=O)C3=C(CC(C)(C)C1(CC=C)C3(C)C)C(=O)c1ccccc1C2=O</smiles>

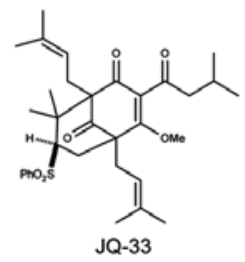

JQ-34<smiles>[2H]C1=C(O)C(CC=C(C)C)(CC(CO)=C(C)C)C(O)=C(CC=C(C)C)C1=O</smiles>

BM006<smiles>CC(C)=CCC1=C(O)C2(CC=C(C)C)CCC3CC(=O)C1(C)C(=O)C32C</smiles>

BM008

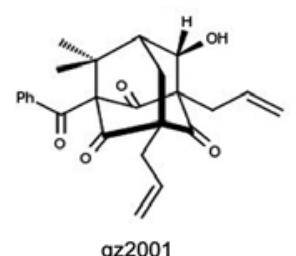<smiles>CC(C)=CCC1=C(O)[C@](C)(CC=C(C)C)C(=O)C(CC=C(C)C)(CC=C(C)C)C1=O</smiles>

BM002

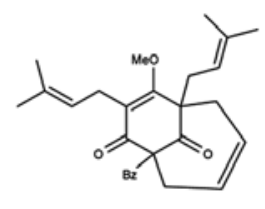

BM007

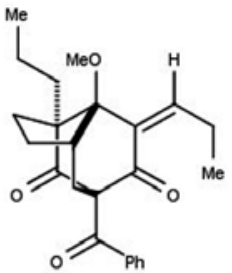

BM-01-1013F2

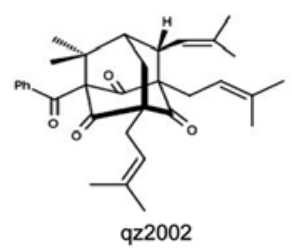<smiles>CC(C)=CCOC(=O)OC1=C(C)C(=O)C(CC=C(C)C)=C(C)[C@]1(C)CC=C(C)C</smiles>

BM003

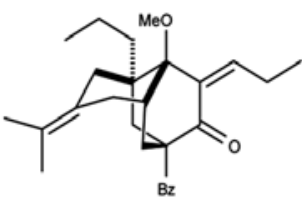

BM01810

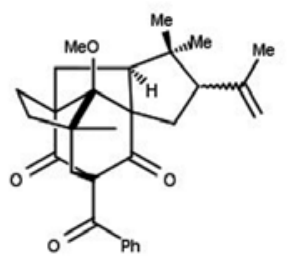

BM-01-1022

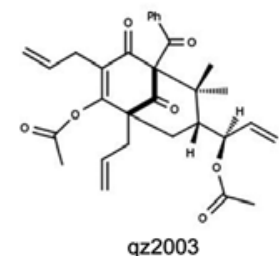<smiles>CC(C)=CCC1=C(O)C(CC=C(C)C)(CC=C(C)C)C(O)=C(Br)C1=O</smiles>

BM004

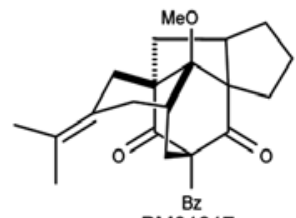

BM01817<smiles>CC(C)=CCC1=C(O)[C@]2(C)CC=CC2(CC=C(C)C)C(=O)[C@]1(C)CC=C(C)C</smiles>

BM005

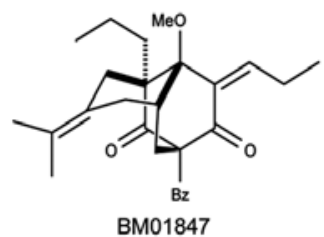

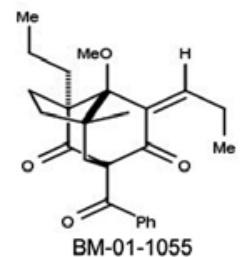<smiles>C=C(C)C1CC2=C(O)C(CC=C(C)C)(CC=C(C)C)C(=O)C(C(C)=O)=C2O1</smiles>

BM-01-1011

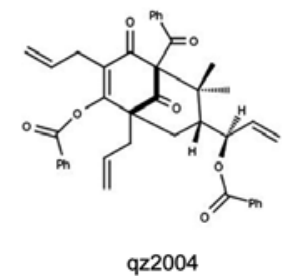

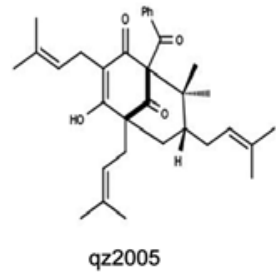

Figure 1. Synthesized and screened compounds. A panel of synthesized analogues of the type B PPAP natural product clusianone and the type A PPAP natural product nemorosone. The compounds were synthesized with a procedure involving tandem alkylative dearomatization/annulation of acylphloroglucinols or employing $\mathrm{Mn}(\mathrm{III}) / \mathrm{Cu}(\mathrm{II})$-mediated oxidative radical cyclizations of dearomatized phloroglucinol substrates. 


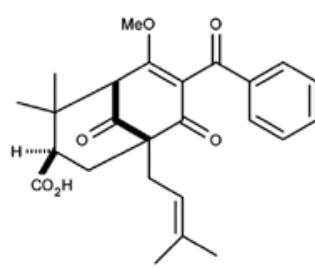

\section{JQ-101}

$\mathrm{IC}_{50}: 30 \mu \mathrm{M}$

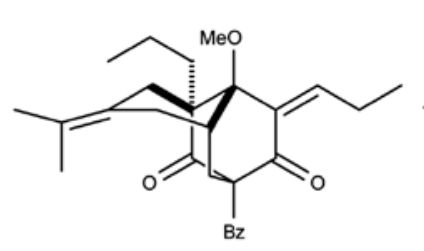

BM 1810

$\mathrm{IC}_{50}: 70 \mu \mathrm{M}$

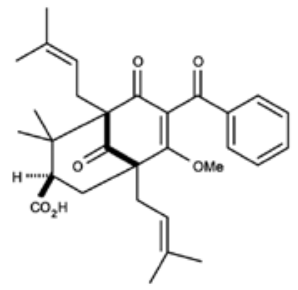

JQ-101

$\mathrm{IC}_{50}: 30 \mu \mathrm{M}$

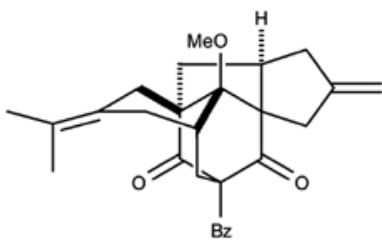

BM1817

$\mathrm{IC}_{50}: 80 \mu \mathrm{M}$

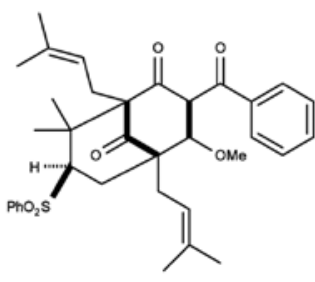

JQ-03

$\mathrm{IC}_{50}: 60 \mu \mathrm{M}$

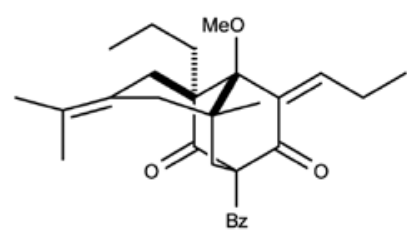

BM 1847

$\mathrm{IC}_{50}: 90 \mu \mathrm{M}$

Figure 2. Compounds with SIRT1 inhibitory activity. A biochemical-based inhibitory assay with recombinant SIRT1 and SIRT2 was performed. Five compounds show SIRT1 inhibition activity with $\mathrm{IC}_{50}$ from 30 to $90 \mu \mathrm{M}$.

placed in the upper chamber coated with matrigel and $500 \mu 1$ of culture media was added to the lower chamber of the transwell. After $48 \mathrm{~h}$ of incubation at $37^{\circ} \mathrm{C}$, the transwells were removed from the 24-well plates and stained with Diff-Quick solution. The invading cells were enumerated by microscopy.

\section{Results}

Design, synthesis and evaluation of SIRT1 inhibitors based on polyprenylated acylphloroglucinol (PPAP) natural products. Polyprenylated acylphloroglucinols (PPAPs) are a family of natural products that possess a wide range of different biological activities (44). There are more than 100 PPAPs discovered to date. Most of these were isolated from plants and trees from Clusiaceae (Guttiferae). PPAPs are divided into three types (A, B and C), depending on the position of the acyl group on the bicyclic core (44). It has been reported that the PPAPs guttiferone $\mathrm{G}$ and aristoforin have SIRT1 inhibitory activity, although little biological function has been shown (39). We synthesized a panel of derivatives of PPAP compounds using our reported procedure involving tandem alkylative dearomatization/annulation of acylphloroglucinols to rapidly construct the bicyclo[3.3.1] nonane-1,3,5-trione core (41) or employing $\mathrm{Mn}(\mathrm{III}) / \mathrm{Cu}(\mathrm{II})$-mediated oxidative radical cyclizations of dearomatized phloroglucinol substrates (42).

Using a fluorogenic substrate, we performed a biochemical-based inhibitory assay with recombinant SIRT1 and SIRT2. We synthesized and screened over 35 synthesized analogues of the type B PPAP natural product clusianone and the type A PPAP natural product nemorosone (see Fig. 1 for details and structures). Among the synthesized PPAP analogues, we identified five compounds with SIRT1 inhibitory activity with $\mathrm{IC}_{50}$ ranging from 30 to $90 \mu \mathrm{M}$, including JQ-101, JQ-3, BM1810, BM1817 and BM1847 (Fig. 2). One of these compounds, designated JQ-101, which showed the best in vitro activity for inhibition of SIRT1, forms the major focus of this report.

Inhibition of SIRT1 deacetylase activity by JQ-101 in vitro and in vivo. Using a fluorogenic substrate, we performed biochemical-based inhibition assays with recombinant SIRT1 and SIRT2. JQ-101 inhibited SIRT1 deacetylase activity with an $\mathrm{IC}_{50}$ of $30 \mu \mathrm{M}$ (Fig. 3A and B). JQ-101 also inhibited the closely-related class III HDAC SIRT2, with an $\mathrm{IC}_{50}$ of $150 \mu \mathrm{M}$ (Fig. 3C). Thus, JQ-101 has 5-fold selectivity in inhibiting SIRT1 over SIRT2. Sirtinol was used as a positive control for the assay, with an $\mathrm{IC}_{50}$ value of $60 \mu \mathrm{M}$ for SIRT1 (Fig. 3B) and $20 \mu \mathrm{M}$ for SIRT2 (Fig. 3C), respectively, in good agreement with reported values.

We next examined whether JQ-101 can target SIRT1 in a cell-based system. It is well established that lysine 382 of p53 is a cellular substrate of SIRT1 (46). We therefore assessed the levels of lysine 382-acetylated p53 in p53 wild-type cells exposed to JQ-101 compared to vehicle. Treatment with JQ-101 increased the acetylation levels of p53 in LNCaP and H460 cells (Fig. 3D and E). We also assessed the level of histone $\mathrm{H} 4$ acetylation at lysine 16 , another confirmed substrate for SIRT1. JQ-101 treatment increased the levels of histone H4 acetylation in both H460 (p53 wt) and H1299 (p53 null) cells (Fig. 3E and F). These findings suggest that JQ-101 can prevent the deacetylation of known biological substrates of SIRT1, thereby demonstrating on-target SIRT1 inhibitory activity of JQ-101 in vivo.

JQ-101 suppresses cancer cells growth. Several SIRT1 inhibitors have been shown to produce antitumor activity in vitro and in mouse xenograft models. To investigate the effects of JQ-101 on cancer cell growth and survival inhibition, we determined the inhibitory ability of JQ-101 on a panel of human cancer cell lines, with normal human cell lines as 


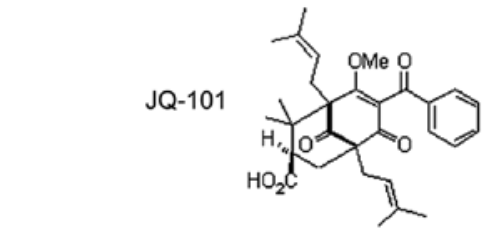

B

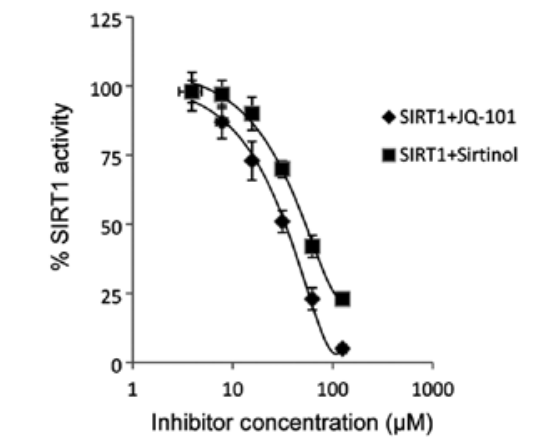

D

D

p53

$\beta$-actin
A

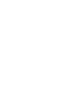

E

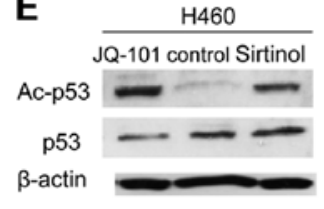

\&

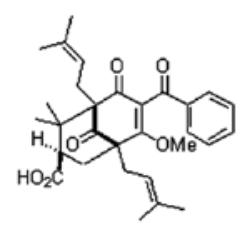

C
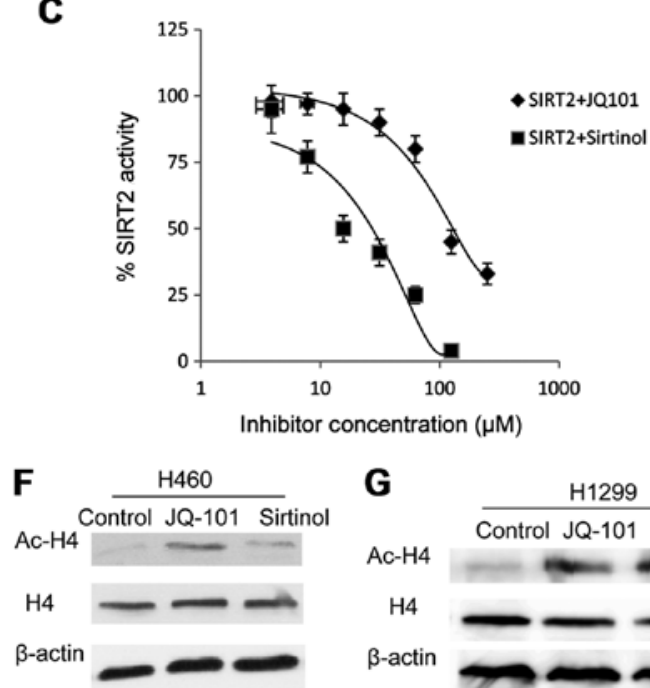

G

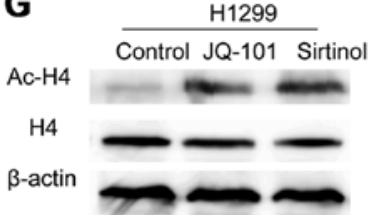

Figure 3. Identification of JQ-101 as a selective SIRT1 inhibitor.(A) Chemical structure of JQ-101. (B and C) In vitro SIRT1 and SIRT2 inhibition assays. Recombinant human SIRT1 and SIRT2 were exposed to various concentrations of JQ-101 and sirtinol as indicated, and their relative inhibitory potential (against DMSO as vehicle control) was analyzed and displayed as a percentage of SIRT1 activity. JQ-101 inhibited recombinant SIRT1 and SIRT2 deacetylase activity at an IC ${ }_{50}$ of 30 and $150 \mu \mathrm{M}$, respectively. Sirtinol was included as a positive control. The $\mathrm{IC}_{50}$ determination of JQ-101 was performed using GraphPad Prism 6.0. Each point represents the mean of three independent experiments; error bars indicate SD. (D and E) JQ-101 increases levels of lysine 382 acetylated p53. The human prostate cancer cell line (D) LNCaP and (E) H460 were treated with JQ-101 at $50 \mu \mathrm{M}$ or sirtinol at $50 \mu \mathrm{M}$ or vehicle control for $2 \mathrm{~h}$. Cell lysates were prepared and immunoblotted with anti-Ac-p53, p53 or $\beta$-actin antibodies. (F and G) JQ-101 increased levels of histone H4 acetylated at lysine 16 . (F) H460 and (G) H1299 cells were treated with JQ-101 at $50 \mu \mathrm{M}$ or sirtinol at $50 \mu \mathrm{M}$ or vehicle control for $2 \mathrm{~h}$. Cell lysates were prepared and immunoblotted with anti-Ac-H4K16, anti-H4 or $\beta$-actin antibodies.

serving as non-cancer controls. As shown in Fig. 4A and B, JQ-101 suppressed LNCaP and H460 tumor cell growth in a dose-dependent manner. We screened eight different cancer cell lines in total, including lines derived from prostate cancers (LNCaP and PC3), lung cancers (H460 and A549), leukemia (Jurkat), lymphoma (Ramos), and breast cancers (ZR75 and MDA231). JQ-101 suppressed tumor cell growth at $\mathrm{IC}_{50}$ values ranging from 20 to $119 \mu \mathrm{M}$ (Table I). By comparison, JQ-101 was much less toxic in normal human cells, including one normal human prostate epithelial cell line (PZ-HPV-7) and one human lung fibroblast cell line (MRC-5) (Table I). These results suggest that JQ-101 exerts cancer cell-selectivity in inhibiting the growth of cells.

To more specifically study the role of SIRT1 in control of cancer cell growth, SIRT1 was knocked down with SIRT1 RNAi in LNCaP and H460 cells. SIRT1 silencing resulted in cancer cell growth suppression (Fig. 4C). This suggests that SIRT1 is important in control of growth in these cancer cells. To further study the specificity of JQ-101 mediated cancer cell growth, we treated the both SIRT1-silenced and vector control-transfected H460 cancer cell lines with JQ-101. The effect of JQ-101 on cancer cell growth is greatly attenuated in the SIRT1-silenced cells (Fig. 4D). This suggests that JQ-101 suppresses cancer cell growth by targeting SIRT1.

JQ-101 induces both apoptosis and cell senescence. To further elucidate the molecular mechanisms of this tumor- selective growth suppression, Annexin V and $\beta$-gal staining were carried out to study the effects of JQ-101 in inducing apoptosis or cell senescence. JQ-101 treatment induced cell death and apoptosis in LNCaP cells; proportions of cells in both late stage (14.5\%) and early stage (3.2\%) apoptosis increased after JQ-101 treatment (Fig. 5A). In contrast, apoptosis was not observed in H1299 cells (data not shown); instead, JQ-101 treatment induced cell senescence in H1299 cells (Fig. 5B) but not in LNCaP cells (data not shown). This suggests that the induction of either apoptosis or cell senescence, in a context-dependent fashion, are the mechanisms whereby JQ-101 suppresses cancer cell growth and survival.

JQ-101 inhibits cancer cell invasion. Recent studies have shown that SIRT1 plays an important role in cancer cell migration and invasion $(14,16,46)$. We therefore determined whether SIRT1 inhibition by JQ-101 could inhibit cancer cell invasion. PC3 prostate cancer and A549 lung cancer cells, which exhibit invasive behavior, were exposed to JQ-101, and the effects on cancer cell invasion were quantitated, using a Transwell chamber assay system with matrigel coated on the top of an ECM-like membrane to prevent the transmigration of non-invasive cells. Exposure to JQ-101 significantly decreased invasion compared to vehicle control, producing a 3.5-fold reduction in PC3 cell invasion and a 4.2-fold reduction in A549 cells (Fig. 6A and B), suggesting that JQ-01 exerts an inhibitory effect on cancer cell invasion. 
A

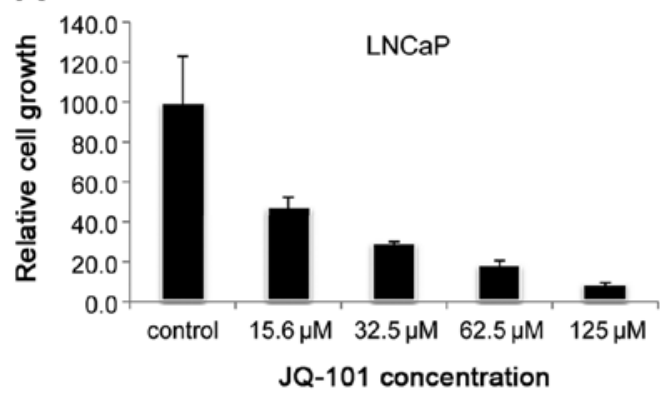

B

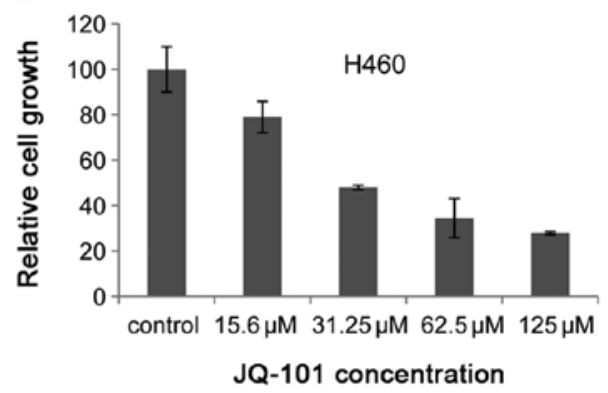

C

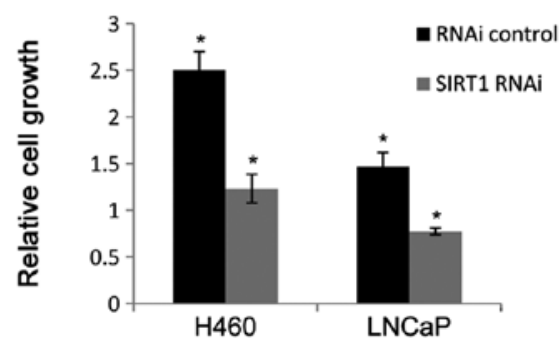

D

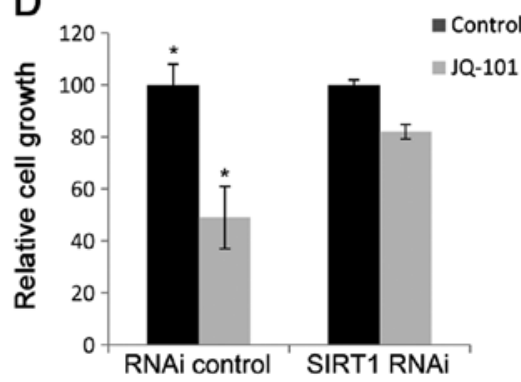

$\mathrm{E}$

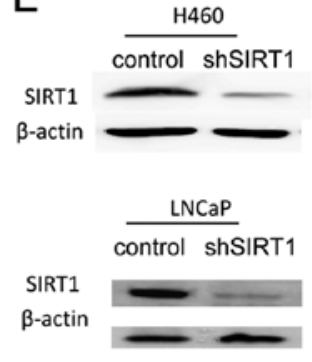

Figure 4. JQ-101 suppresses tumor cells growth and survival. (A and B) Cells from the prostate cancer line LNCaP or lung cancer line H460 were treated with varying concentrations of JQ-101 as indicated in the graph for $72 \mathrm{~h}$, or vehicle control (DMSO) and MTS assay was performed. The error bars indicate the SEM. (C) SIRT1 silencing suppressed both LNCaP and $\mathrm{H} 460$ cell growth and survival. A total of 2x10 ${ }^{4}$ SIRT-silenced or shRNA-control LNCaP or H460 cells were seeded onto 24-well plates and counted after $72 \mathrm{~h}$ of culture. The error bars indicate the SEM. (D) SIRT1 knockdown reduces the inhibitory effects of JQ-101 on H460 proliferation. A total of 2x10 4 SIRT-silenced or shRNA-control H460 cells were seeded onto 24-well plates, treated with $50 \mu \mathrm{M} \mathrm{JQ}-101$ for 3 days and viable cells were enumerated. The error bars indicate the SEM. Asterisks indicate significant differences between experimental versus control group $\left({ }^{*} \mathrm{p}<0.05\right)$. (E) Immunoblot analysis shows the SIRT1 levels in the LNCap and H460 knockdown cells that were used for the cell proliferation analysis in (C and D).

A

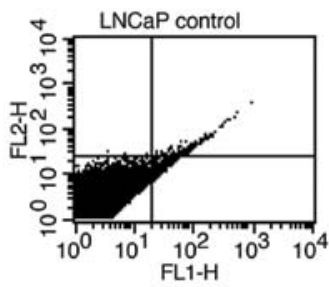

Quadrant Statistics

\begin{tabular}{cr} 
Quad & $\%$ Total \\
\hline UL & 0.10 \\
UR & 2.02 \\
LL & 92.56 \\
LR & 5.32
\end{tabular}

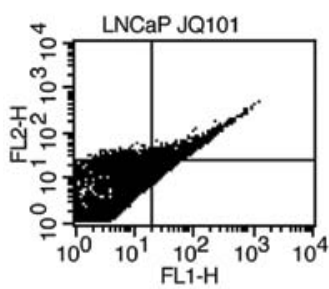

B
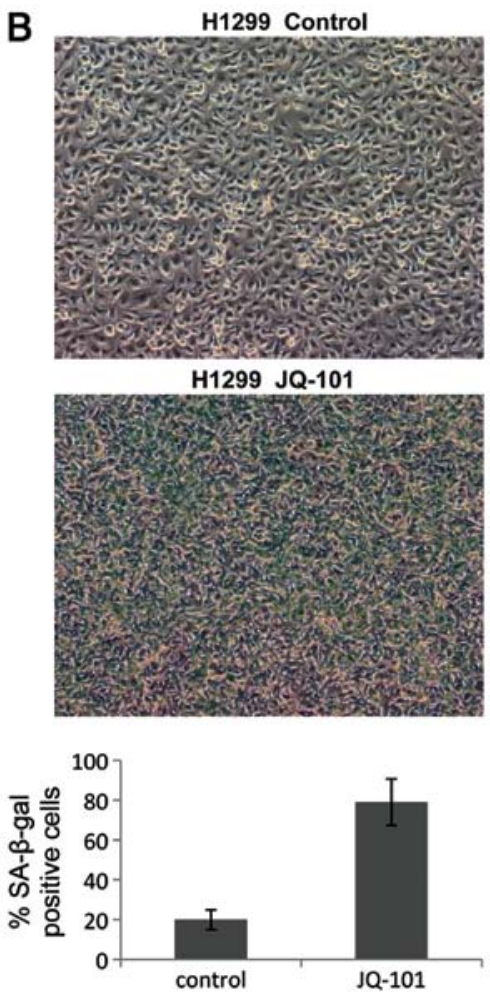

Figure 5. JQ-101 induces either apoptosis or cell senescence. (A) JQ-101 induces apoptosis in prostate LNCaP cells. LNCaP cells were treated with JQ-101 (50 $\mu$ M) or vehicle control for $72 \mathrm{~h}$. The cells were collected, labeled with Annexin V antibody/PI, and analyzed with flow cytometry. UL, dead cells; UR, late apoptosis; LL, live cells; LR, early apoptosis. (B) The lung cancer cell lines $\mathrm{H} 460$ was seeded at approximately $50 \%$ confluence and exposed to JQ-101 at $50 \mu \mathrm{M}$ for 3 days. $\mathrm{X}$-Gal-based $\beta$-galactosidase staining was performed. The $\beta$-galactosidase staining-positive cells were quantitated by microscopy and the percentage of SA- $\beta$-Gal positive cells was calculated. The histograms represent the mean $\pm \mathrm{SD}$ of triplicates. Asterisks indicate significant differences between two groups ( $\mathrm{p}<0.01)$. 
A
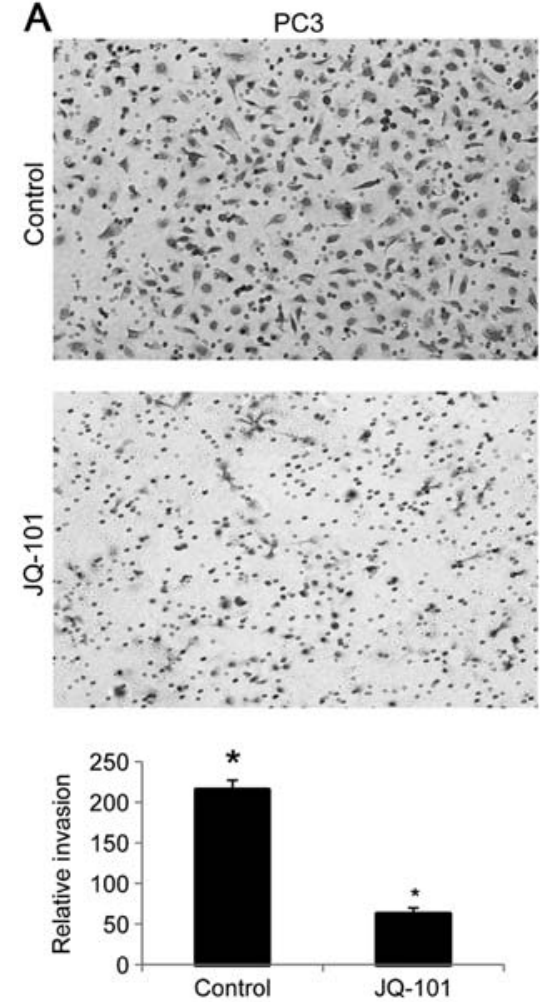

B
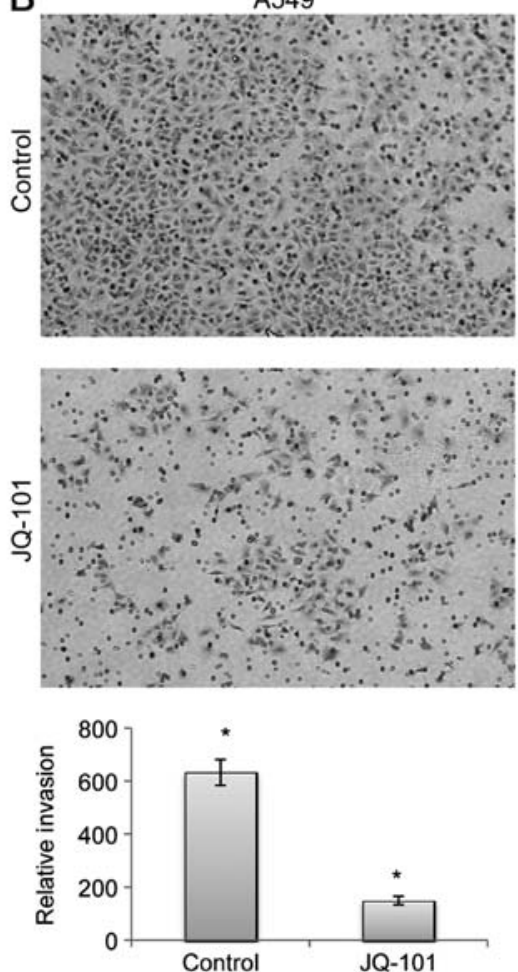

Figure 6. JQ-101 decreases cancer cell invasion. A total of $2.5 \times 10^{4}$ (A) PC3 or (B) A549 cells in $500 \mu 1$ medium mixed with JQ-101 (50 $\mu$ M) or vehicle were loaded onto transwells coated with matrigel. After 48 -h incubation at $37^{\circ} \mathrm{C}$, the transwells were removed from 24 -well plates and stained. The invading cells were quantitated by light microscopy. A representative experiment from three independent experiments is shown. Asterisks indicate significant differences between two groups $\left({ }^{*} \mathrm{p}<0.05\right)$.

\section{Discussion}

SIRT1 has attracted much attention for its functional roles in cancer (47). We report here a new SIRT1 inhibitor JQ-101, a compound possessing a polyprenylated acylphloroglucinol (PPAP) core, which exhibits selective inhibition of tumor cell line growth and survival though induction of apoptosis and cellular senescence, and significantly suppresses cancer cell invasion.

To date, several SIRT1 inhibitors have been developed with a broad range of potency in SIRT1 inhibition (32-36). The $\mathrm{IC}_{50}$ for JQ-101 against STIR1 is approximately $30 \mu \mathrm{M}$, a potency similar to several of the previously reported SIRT1inhibitory compounds, including sirtinol (31), cambinol (32), salermide (37), tenovin-6 (34) and splitomycin (35). Most of the SIRT1 inhibitors identified previously also possess SIRT2 inhibitory activity, due to the high level of sequence similarity between SIRT1 and SIRT2. While JQ-101 also exhibited inhibitory effects on SIRT2, 5-fold higher concentrations were necessary, making JQ-101 somewhat SIRT1-specific. Our results show that JQ-101 can inhibit the growth and survival of a panel of cancer cell lines. Although we cannot rule out the possibility that minor inhibitory effects of SIRT 2 activity may contribute in part to the observed selective cytotoxicity of JQ-101, these studies were carried out under conditions far below the $\mathrm{IC}_{50}$ for SIRT2. In addition, our results show that SIRT1 knockdown greatly impairs the effect of JQ-101 on cell growth and survival (Fig. 4D), demonstrating that SIRT1 is the major target of JQ-101.
It has been shown that SIRT1 inhibition can induce apoptosis in many cancer cell types, in particular p53-wild-type cells (45,48-50); alternatively, SIRT1 inhibition can also induce cell senescence (51-53). Our results show that JQ-101 can trigger either apoptosis in p53-wild-type LNCaP cells or induce cell senescence in p53-null H1299 cells (Fig. 5). Depending upon the specific genetic background of the cancer cell, SIRT1 inhibition by JQ-101 may utilize different downstream targets of SIRT1 to induce either apoptosis or cell senescence, to suppress cancer cell growth and survival.

We also show in this study that JQ-101 treatment can greatly reduce cancer cell invasion (Fig. 6). We have previously identified SIRT1 as a positive regulator of epithelial-tomesenchymal transition (EMT), cell invasion, and metastatic growth of prostate cancer cells, and put forward SIRT1 as a potential therapeutic target to reverse EMT and to prevent prostate cancer progression $(8,19)$. Consistent with our findings, it has been shown that SIRT1 inhibition reduces cancer cell migration and invasion while SIRT1 activation promotes cancer metastasis $(12,15,23,54)$. These results suggest that SIRT1 plays an important role in cancer invasion and metastasis, and that development of SIRT1 inhibitors may represent a critical new targeted strategy to prevent cancer metastasis and progression.

Given our findings that JQ-101 can selectively suppress cancer cell growth by inducing tumor cell apoptosis and senescence, and significantly inhibit cancer cell invasion, these results suggest a potential role of JQ-101 or a derivative in a SIRT1-targeted approach to cancer therapy. Studies are under 
way to delineate the effects of JQ-101 on tumor formation, growth and metastasis in mouse models in vivo. JQ-101 will also serve as a novel chemical scaffold for future development of more potent SIRT1 inhibitors to be used in cancer treatment.

\section{Acknowledgements}

We sincerely thank Dr F. Picard (Laval University, Canada) for providing SIRT1 shRNA vector. We thank Branko Mitasev (Department of Chemistry, Boston University) for compound synthesis, and thank Heba Ijaz, Nadine Aziz, Marina Amaro, and Elizabeth Cho (Cancer Center, Boston University) for their technical support. This study was supported by grants from the National Cancer Institute (CA141036 and CA129046) (Y.D.), (CA101992 and CA164245) (D.V.F.), (GM-073855 and GM-067041) (J.A.P.Jr), the Department of Defense (PC100093) (D.V.F.), National Science Foundation (CHE-0848082) (J.A.P.Jr), the Clinical and Translational Science Institute award of NIH (UL1RR025771) (Y.D.), and the Department of Medicine Pilot Project Grant (Y.D.).

\section{References}

1. Bordone L and Guarente L: Calorie restriction, SIRT1 and metabolism: understanding longevity. Nat Rev Mol Cell Biol 6: 298-305, 2005.

2. Inoue T, Hiratsuka M, Osaki $M$ and Oshimura $M$ : The molecular biology of mammalian SIRT proteins: SIRT2 in cell cycle regulation. Cell Cycle 6: 1011-1018, 2007.

3. Saunders LR and Verdin E: Sirtuins: critical regulators at the crossroads between cancer and aging. Oncogene 26: 5489-5504, 2007.

4. Donmez G and Guarente L: Aging and disease: connections to sirtuins. Aging Cell 9: 285-290, 2010.

5. Bosch-Presegue L and Vaquero A: The dual role of sirtuins in cancer. Genes Cancer 2: 648-662, 2011.

6. Knight JR and Milner J: SIRT1, metabolism and cancer. Curr Opin Oncol 24: 68-75, 2012.

7. Chu F, Chou PM, Zheng X, Mirkin BL and Rebbaa A: Control of multidrug resistance gene mdr1 and cancer resistance to chemotherapy by the longevity gene sirt1. Cancer Res 65: 10183-10187, 2005.

8. Kojima K, Ohhashi R, Fujita Y, et al: A role for SIRT1 in cell growth and chemoresistance in prostate cancer PC3 and DU145 cells. Biochem Biophys Res Commun 373: 423-428, 2008.

9. Lee $\mathrm{SM}, \mathrm{Bae} \mathrm{JH}, \mathrm{Kim} \mathrm{MJ}$, et al: Bcr-Abl-independent imatinib-resistant K562 cells show aberrant protein acetylation and increased sensitivity to histone deacetylase inhibitors. J Pharmacol Exp Ther 322: 1084-1092, 2007.

10. Liang XJ, Finkel T, Shen DW, Yin JJ, Aszalos A and Gottesman MM: SIRT1 contributes in part to cisplatin resistance in cancer cells by altering mitochondrial metabolism. Mol Cancer Res 6: 1499-1506, 2008.

11. Marshall GM, Liu PY, Gherardi S, et al: SIRT1 promotes $\mathrm{N}$-Myc oncogenesis through a positive feedback loop involving the effects of MKP3 and ERK on N-Myc protein stability. PLoS Genet 7: e1002135, 2011.

12. Suzuki K, Hayashi R, Ichikawa T, et al: SRT1720, a SIRT1 activator, promotes tumor cell migration, and lung metastasis of breast cancer in mice. Oncol Rep 27: 1726-1732, 2012.

13. Gabr AG, Goto H, Hanibuchi M, et al: Erlotinib prevents experimental metastases of human small cell lung cancer cells with no epidermal growth factor receptor expression. Clin Exp Metastasis 29: 207-216, 2012

14. Byles V, Zhu L, Lovaas JD, et al: SIRT1 induces EMT by cooperating with EMT transcription factors and enhances prostate cancer cell migration and metastasis. Oncogene 31: 4619-4629, 2012.

15. Zhang Y, Zhang M, Dong H, et al: Deacetylation of cortactin by SIRT1 promotes cell migration. Oncogene 28: 445-460, 2009.

16. Nakane K, Fujita Y, Terazawa R, et al: Inhibition of cortactin and SIRT1 expression attenuates migration and invasion of prostate cancer DU145 cells. Int J Urol 19: 71-79, 2012.
17. Holloway KR, Calhoun TN, Saxena M, et al: SIRT1 regulates Dishevelled proteins and promotes transient and constitutive Wnt signaling. Proc Natl Acad Sci USA 107: 9216-9221, 2010.

18. Lovaas JD, Zhu L, Chiao CY, Byles V, Faller DV and Dai Y: SIRT1 enhances matrix metalloproteinase-2 expression and tumor cell invasion in prostate cancer cells. Prostate 73: 522-530, 2013.

19. Potente M, Ghaeni L, Baldessari D, et al: SIRT1 controls endothelial angiogenic functions during vascular growth. Genes Dev 21: 2644-2658, 2007.

20. Potente $M$ and Dimmeler S: Emerging roles of SIRT1 in vascular endothelial homeostasis. Cell Cycle 7: 2117-2122, 2008.

21. Jang KY, Hwang SH, Kwon KS, et al: SIRT1 expression is associated with poor prognosis of diffuse large B-cell lymphoma. Am J Surg Pathol 32: 1523-1531, 2008.

22. Huffman DM, Grizzle WE, Bamman MM, et al: SIRT1 is significantly elevated in mouse and human prostate cancer. Cancer Res 67: 6612-6618, 2007.

23. Zhao G, Cui J, Zhang JG, et al: SIRT1 RNAi knockdown induces apoptosis and senescence, inhibits invasion and enhances chemosensitivity in pancreatic cancer cells. Gene Ther 18: 920-928, 2011.

24. Cha EJ, Noh SJ, Kwon KS, et al: Expression of DBC1 and SIRT1 is associated with poor prognosis of gastric carcinoma. Clin Cancer Res 15: 4453-4459, 2009.

25. DiMeo TA, Anderson K, Phadke P, et al: A novel lung metastasis signature links Wnt signaling with cancer cell self-renewal and epithelial-mesenchymal transition in basal-like breast cancer. Cancer Res 69: 5364-5373, 2009.

26. Lee H, Kim KR, Noh SJ, et al: Expression of DBC1 and SIRT1 is associated with poor prognosis for breast carcinoma. Hum Pathol 42: 204-213, 2011.

27. Chen J, Zhang B, Wong N, et al: Sirtuin 1 is upregulated in a subset of hepatocellular carcinomas where it is essential for telomere maintenance and tumor cell growth. Cancer Res 71: 4138-4149, 2011.

28. Kriegl L, Vieth M, Kirchner T and Menssen A: Up-regulation of c-MYC and SIRT1 expression correlates with malignant transformation in the serrated route to colorectal cancer. Oncotarget 3: 1182-1193, 2012.

29. Tseng RC, Lee CC, Hsu HS, Tzao C and Wang YC: Distinct HIC1-SIRT1-p53 loop deregulation in lung squamous carcinoma and adenocarcinoma patients. Neoplasia 11: 763-770, 2009.

30. Bitterman KJ, Anderson RM, Cohen HY, Latorre-Esteves M and Sinclair DA: Inhibition of silencing and accelerated aging by nicotinamide, a putative negative regulator of yeast sir 2 and human SIRT1. J Biol Chem 277: 45099-45107, 2002.

31. Grozinger CM, Chao ED, Blackwell HE, Moazed D and Schreiber SL: Identification of a class of small molecule inhibitors of the sirtuin family of NAD-dependent deacetylases by phenotypic screening. J Biol Chem 276: 38837-38843, 2001.

32. Heltweg B, Gatbonton T, Schuler AD, et al: Antitumor activity of a small-molecule inhibitor of human silent information regulator 2 enzymes. Cancer Res 66: 4368-4377, 2006.

33. Solomon JM, Pasupuleti R, Xu L, et al: Inhibition of SIRT1 catalytic activity increases p53 acetylation but does not alter cell survival following DNA damage. Mol Cell Biol 26: 28-38, 2006.

34. Lain S, Hollick JJ, Campbell J, et al: Discovery, in vivo activity, and mechanism of action of a small-molecule p53 activator. Cancer Cell 13: 454-463, 2008.

35. Bedalov A, Gatbonton T, Irvine WP, Gottschling DE and Simon JA: Identification of a small molecule inhibitor of Sir2p. Proc Natl Acad Sci USA 98: 15113-15118, 2001.

36. Choi G, Lee J, Ji JY, et al: Discovery of a potent small molecule SIRT1/2 inhibitor with anticancer effects. Int J Oncol 43: 1205-1211, 2013.

37. Lara E, Mai A, Calvanese V, et al: Salermide, a Sirtuin inhibitor with a strong cancer-specific proapoptotic effect. Oncogene 28: 781-791, 2009.

38. Suzuki T, Imai K, Nakagawa $\mathrm{H}$ and Miyata N: 2-Anilinobenzamides as SIRT inhibitors. ChemMedChem 1: 1059-1062, 2006.

39. Gey C, Kyrylenko S, Hennig L, et al: Phloroglucinol derivatives guttiferone $\mathrm{G}$, aristoforin, and hyperforin: inhibitors of human sirtuins SIRT1 and SIRT2. Angew Chem Int Ed Engl 46: 5219-5222, 2007.

40. Zhang Y, Zhang Q, Zeng SX, Zhang Y, Mayo LD and Lu H: Inauhzin and Nutlin3 synergistically activate p53 and suppress tumor growth. Cancer Biol Ther 13: 915-924, 2012. 
41. Qi J and Porco JA Jr: Rapid access to polyprenylated phloroglucinols via alkylative dearomatization-annulation: total synthesis of (+/-)-clusianone(1). J Am Chem Soc 129: 12682-12683, 2007.

42. Mitasev B and Porco JA Jr: Manganese(III)-mediated transformations of phloroglucinols: a formal oxidative $[4+2]$ cycloaddition leading to bicyclo[2.2.2] octadiones. Org Lett 11: 2285-2288, 2009.

43. Zhang Q and Porco JA Jr: Total synthesis of (+/-)-7-epi-nemorosone. Org Lett 14: 1796-1799, 2012.

44. Biber N, Mows K and Plietker B: The total synthesis of hyperpapuanone, hyperibone L, epi-clusianone and oblongifolin A. Nat Chem 3: 938-942, 2011.

45. Vaziri H, Dessain SK, Ng Eaton E, et al: hSIR2(SIRT1) functions as an NAD-dependent p53 deacetylase. Cell 107: 149-159, 2001.

46. Noh SJ, Baek HA, Park HS, et al: Expression of SIRT1 and cortactin is associated with progression of non-small cell lung cancer. Pathol Res Pract 209: 365-370, 2013.

47. Liu T, Liu PY and Marshall GM: The critical role of the class III histone deacetylase SIRT1 in cancer. Cancer Res 69 1702-1705, 2009.

48. Li L, Wang L, Li L, et al: Activation of $\mathrm{p} 53$ by SIRT1 inhibition enhances elimination of CML leukemia stem cells in combination with imatinib. Cancer Cell 21: 266-281, 2012.
49. Kalle AM, Mallika A, Badiger J, Alinakhi, Talukdar P and Sachchidanand: Inhibition of SIRT1 by a small molecule induces apoptosis in breast cancer cells. Biochem Biophys Res Commun 401: 13-19, 2010.

50. Sun Y, Sun D, Li F, et al: Downregulation of Sirt1 by antisense oligonucleotides induces apoptosis and enhances radiation sensitization in A549 lung cancer cells. Lung Cancer 58: 21-29, 2007.

51. Ota H, Tokunaga E, Chang K, et al: Sirt1 inhibitor, Sirtinol, induces senescence-like growth arrest with attenuated Ras-MAPK signaling in human cancer cells. Oncogene 25: 176-185, 2006.

52. Huang J, Gan Q, Han L, et al: SIRT1 overexpression antagonizes cellular senescence with activated ERK/S6k1 signaling in human diploid fibroblasts. PLoS One 3: e1710, 2008.

53. Jang SY, Kim SY and Bae YS: p53 deacetylation by SIRT1 decreases during protein kinase CKII downregulation-mediated cellular senescence. FEBS Lett 585: 3360-3366, 2011.

54. Saxena M, Dykes SS, Malyarchuk S, Wang AE, Cardelli JA and Pruitt K: The sirtuins promote Dishevelled-1 scaffolding of TIAM1, Rac activation and cell migration. Oncogene: Dec 23, 2013 (Epub ahead of print). 